\title{
Wer's glaubt: Orthopäden kraftvoll und klug zugleich
}

\begin{abstract}
Was Sie aufgrund eigener Erfahrungen nie vermutet hätten, wird nun wissenschaftlich bestätigt: Männliche Orthopäden haben nicht nur mehr Muskeln als Anästhesisten, sie sind auch noch intelligenter.
\end{abstract}

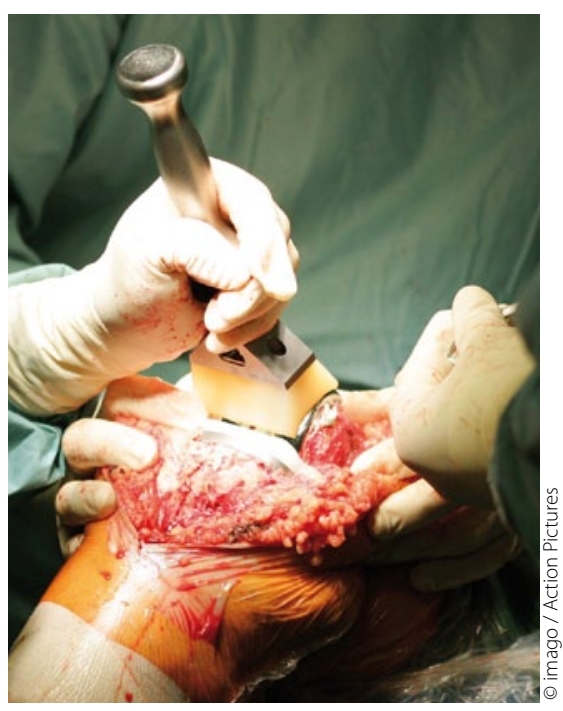

Orthopäden: Hier wohnt ein gesunder Geist in einem gesunden Körper.
— In der medizinischen Literatur, im Internet und in vielen bissig bis witzig gemeinten Alltagsbemerkungen im OP werden Orthopäden gerne in die Nähe von Primaten gerückt. Manche halten die Kombination aus brutaler Kraft und Ignoranz geradezu für eine Voraussetzung, um Orthopäde zu werden: „Typisch Orthopäde, stark wie ein Ochse, aber nur halb so intelligent". Mit diesem Vorurteil sollte nun in einer multizentrischen prospektiven Vergleichsstudie in drei englischen Krankenhäusern der dritten Versorgungsstufe aufgeräumt werden.

36 operierende Orthopäden und 40 männliche Anästhesisten in der hierarchischen Stellung von Chefärzten oder Oberärzten unterzogen sich einem Intelligenztest und einer Messung der Griffstärke der dominanten Hand. Erwartungsgemäß hatten die Orthopäden eine statistisch signifikant größere Griffstärke als die Anästhesisten (47,25 $\pm 6,95 \mathrm{~kg}$ vs. $43,83 \pm 7,57 \mathrm{~kg}$ ). Womit aber niemand gerechnet hatte, war das
Ergebnis beim Intelligenztest. Auch hier erreichten die Orthopäden einen statistisch signifikant besseren Wert als die Anästhesisten: 105,19 $\pm 10,85$ im Vergleich zu 98,38 $\pm 14,45$.

\section{Kommentar}

Gerade durch die intellektuell herausfordernde stundenlange Beschäftigung mit Kreuzworträtseln und Sudoku hatte man sich ein besseres Abschneiden der Anästhesisten beim IQ erwartet. Es könnte aber auch sein, dass im Sinne der Hand-Hirn-Theorie präzises Bohren, Hämmern und Sägen eine größere intellektuelle Schulung und Herausforderung darstellt, als das Ablesen von Werten auf Instrumenten. Trotz der zweifellos vorhandenen methodischen Mängel gebührt der Untersuchung das Verdienst, mit einem verbreiteten Vorurteil in wissenschaftlich exakter Weise ein für alle Mal aufgeräumt zu haben.

- P. Subramanian et al.

(Korres.:drpad@hotmail.com): As strong as an ox and twice as clever? Brit. Med. J. 2011; 343: d7506

\section{Wie sich Gesundheitsbehörden die Suppe versalzen}

\section{Essen die Beschäftigten hollän- discher Gesundheitsbehörden in den betriebseigenen Kantinen, nehmen Sie täglich etwa 15,4 g Kochsalz zu sich, womit sie nicht nur gegen ihre eigenen Empfehlungen verstoßen, sondern sich auch einem erhöhten Risiko einer vorzeitigen kardiovas- kulären Mortalität zwischen 23 und $36 \%$ aussetzen.}

- Man sollte annehmen, dass Gesundheitspolitiker und Krankenhausverwaltungen höhere Einsicht darüber besitzen, wie man gesundheitliche Risiken vermeiden kann. An drei zufällig ausgewählten Tagen wurde in 18 Kantinen des holländischen Gesundheitsministeriums, des Gesundheitsrates, der Behörde zur Überwachung der Lebensmittel- sicherheit, von Universitätskrankenhäusern und akademischen Lehrkrankenhäusern der Salzgehalt der Suppen und nicht vegetarischen heißen Mittagsgerichte mit ionenselektiven Elektroden untersucht. Der mittlere Salzgehalt der Gerichte betrug 7,1 $\pm 0,2 \mathrm{~g}$ und überstieg damit bereits mit einer Mahlzeit die tägliche Empfehlung der Salzzufuhr von $6 \mathrm{~g}$. Dieser Wert wurde in allen Kantinen überschritten und lag bei 6,9 $\mathrm{g}$ im Gesundheitsministerium und im Nationalen Gesundheitsrat, 6,0 $\mathrm{g}$ bei der Lebensmittelüberwachungsbehörde, 7,4 g in den Kantinen der Universitätskrankenhäuser und 7,0 $\mathrm{g}$ in den akademischen Lehrkrankenhäusern. Beschäftigte, die in diesen Kantinen aßen, nahmen insgesamt pro Tag 15,4 g Kochsalz zu sich.

\section{Kommentar}

Bei regelmäßigen Essen in ihren Kantinen gehen die Beschäftigten eine Zunahme des Risikos für einen tödlichen Schlaganfall zwischen 23 und $27 \%$ und des Risikos für ein tödliches kardiovaskuläres Ereignis zwischen 23 und 27\% im Vergleich zu Personen mit Beachtung der empfohlenen Kochsalzzufuhr ein. Offensichtlich ist es ein Irrtum anzunehmen, Gesundheitspolitiker würden, trotz der hoffentlich vorhandenen höheren Einsicht, in ihrem engsten Umfeld Erkenntnisse zur Erhaltung der Gesundheit tatsächlich in praktisches Handeln umsetzen.

- Brewster L. M. et al.

(Korres.: I.m.brewster@amc.uva.nl): High salt meals in staff canteens of salt policy makers. Brit. Med. J. 2011; 343: d7352 